\title{
Tuberculous Tonsillitis in a Patient Treated with an Anti-TNF Agent
}

\author{
Sara Ferreira, Paula Vaz-Marques \\ Centro Hospitalar de S. João, Serviço de Medicina Interna, Porto, Portugal
}

Received: 26/06/2017

Accepted: 05/07/2017

Published: $28 / 07 / 2017$

How to cite this article: Ferreira S, Vaz-Marques P. Tuberculous tonsillitis in a patient treated with an anti-TNF agent. EJCRIM 2017;4: doi:10.12890/2017_000690.

Conflicts of Interests: The Authors declare that there are no competing interests.

This article is licensed under a Commons Attribution Non-Commercial 4.0 License

\section{ABSTRACT}

Tuberculosis remains a concern in patients receiving anti-TNF therapy as these individuals have a higher incidence of extrapulmonary and disseminated tuberculosis. Tuberculous tonsillitis is an unusual presentation of extrapulmonary tuberculosis, which is diagnosed mostly in immunodeficient patients. We report the case of a 33-year-old woman, diagnosed with Behçet's syndrome, immunosuppressed with adalimumab, cyclosporine and prednisolone. She had odynophagia for 2 weeks, fever and a hypertrophied, ulcerated left tonsil. A tonsil biopsy revealed a granulomatous inflammatory process. Ziehl-Neelsen staining was positive for acid-fast bacilli. The chest $\mathrm{x}$-ray revealed a milliary pattern. Gastric juice was positive for Mycobacterium tuberculosis complex DNA. A diagnosis of tonsillar and pulmonary tuberculosis was established. This case report highlights the low threshold for a tuberculosis diagnosis in unusual locations in patients treated with antiTNF therapy.

\section{LEARNING POINTS}

- Tuberculosis is a concern in patients receiving anti-TNF therapy as these individuals have a higher incidence of disseminated and extrapulmonary tuberculosis despite previous screening and treatment of latent disease.

- Tuberculous tonsillitis is a rare presentation of extrapulmonary tuberculosis and is thought to occur mostly in immunodeficient patients.

- Tonsillar tuberculosis commonly presents with sore throat and cervical lymphadenopathy which with abnormal tonsillar findings (such as ulcerations, masses and white patches) makes the differential diagnosis with other tonsillar infections or neoplasms challenging.

\section{KEYWORDS}

Tuberculous tonsillitis, adalimumab

\section{CASE DESCRIPTION}

We present the case of a 33-year-old Caucasian woman with a diagnosis of Behçet's syndrome with severe eye disease with left optic neuropathy and bilateral retinal vaso-occlusive vasculitis. The patient had been taking subcutaneous adalimumab 40 mg every 2 weeks (for the last 6 months), cyclosporine $150 \mathrm{mg}$ twice daily (for the last 12 months) and prednisolone 10 mg once daily (for the last 14 months). Before the treatment with adalimumab was initiated, she was evaluated to exclude latent or active tuberculosis: she had no suggestive symptoms or signs, chest x-ray was normal, and an interferon-gamma release assay (IGRA) and two-step tuberculin skin test were both negative. The patient also had arterial hypertension (treated with losartan $50 \mathrm{mg}$ once daily) and a history of migraine.

The patient complained of odynophagia and fever for the last 2 weeks. She had already been treated with several broad-spectrum antibiotics without resolution of her symptoms. The physical examination was unremarkable except for a hypertrophied ulcerated left tonsil (Fig. 1), cervical adenopathy and fever $\left(38^{\circ} \mathrm{C}\right)$. The patient was admitted for further investigation. 
Methods and Procedures

The patient's blood cell count and chemistry were normal, except for an elevated C-reactive protein level ( $40.2 \mathrm{mg} / \mathrm{L}$; normal <3.0 mg/L). A cervical computed tomography (CT) scan was performed which showed hypertrophy of the left tonsil and surrounding tissues, with illdefined boundaries and diffuse hypodensity, prolapsing to the pharyngeal lumen (which was reduced by about one quarter of its diameter) as well as various non-specific bilateral lymphadenopathies. A left tonsil biopsy was performed. The histological examination of the biopsied tissue revealed a granulomatous inflammatory process with necrosis (Fig. 2), while the Ziehl-Neelsen stain of the sample was positive for acid-fast bacilli. Although the patient had no respiratory complaints and a normal lung auscultation, a chest x-ray was performed, which revealed several small and uniformly distributed nodular opacities throughout both lung fields, with a milliary pattern (Fig. 3). Gastric juice collected for examination (as the patient had no respiratory secretions) was positive for Mycobacterium tuberculosis complex DNA.

Adalimumab and cyclosporine were stopped and prednisolone was reduced gradually from 10 to $5 \mathrm{mg} / \mathrm{day}$. The patient initiated antituberculous therapy with a good initial clinical response (persistent apyrexia and improvement of left tonsil hypertrophy). After confirmation that the patient was no longer bacilliferous, she was discharged with antituberculous therapy, prednisolone 5 mg and intraocular injections of triamcinolone (performed by her ophthalmologist). At the time of discharge, the patient was asymptomatic and the chest $x$-ray had also improved. Ocular disease remained stable.

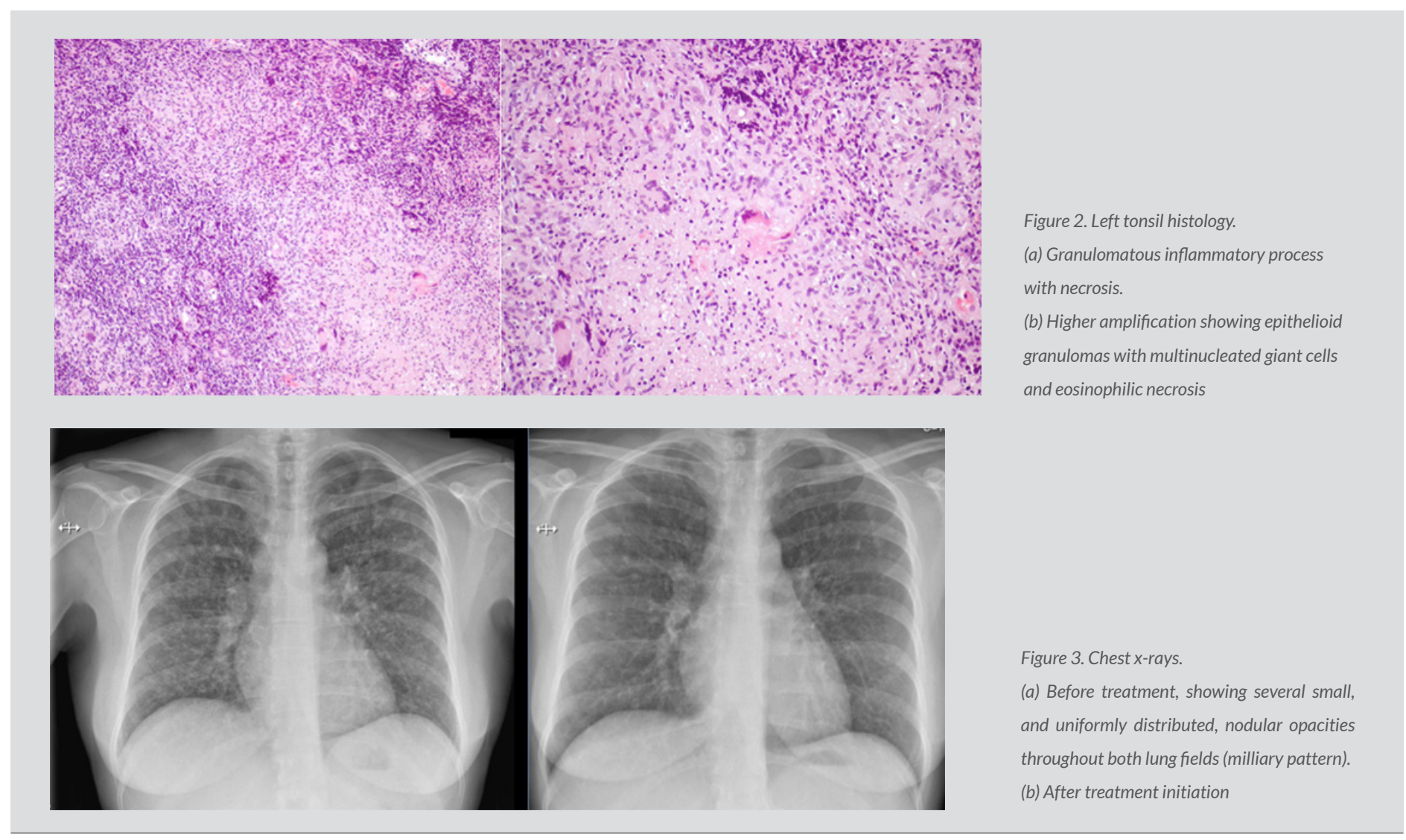




\section{DISCUSSION}

Adalimumab is a fully recombinant human monoclonal antibody that specifically binds with high affinity to human TNF inhibiting its binding to TNF receptors. TNF inhibitors have been shown to decrease inflammation associated with many rheumatological conditions ${ }^{[1]}$. These drugs have recently emerged as new tools for the treatment of non-infectious uveitis, providing additional options for reducing ocular inflammation when long-term corticosteroid-sparing therapy is required. Infliximab and adalimumab have shown encouraging results in multiple trials and are becoming popular for the treatment of ocular inflammation, including in Behçet's disease ${ }^{[2]}$.

The increasingly clinical use of TNF antagonists has improved the management of immunomediated diseases but has also been associated with a higher incidence of infections, especially active tuberculosis. Patients treated with these agents have a higher rate of disseminated and extrapulmonary tuberculosis, despite previous screening and treatment of latent disease ${ }^{[3]}$. Tuberculous tonsillitis is a relatively rare presentation of extrapulmonary tuberculosis and is thought to occur mostly in immunodeficient patients. The diagnosis of tonsillar tuberculosis in the absence of active pulmonary disease is rare since most cases of oral cavity tuberculosis are secondary to pulmonary disease. Occasionally, tonsillar tuberculosis may precede the diagnosis of pulmonary disease. Tonsillar tuberculosis commonly presents with sore throat and cervical lymphadenopathy. This presentation, as well as abnormal tonsillar findings, such as ulcerations, masses and white patches, makes the differential diagnosis with other infections or neoplasm challenging. The diagnosis is based on histopathological findings and identification of tuberculous bacilli ${ }^{[4]}$.

Tuberculosis in patients receiving anti-TNF therapy generally arises from the reactivation of latent infection and usually occurs within the first 3-8 months of treatment. Latent tuberculosis screening, before anti-TNF treatment, is considered essential, given the risk of progression to active tuberculosis and increased susceptibility to more severe forms of the disease. Screening and treatment of latent tuberculosis, prior to initiation of TNF antagonists, has been shown to be effective in markedly reducing the rates of tuberculosis reactivation. Despite these measures, tuberculosis may still occur in those with negative tests, probably sometimes representing new infections instead of reactivations. Screening tests carry a risk of false-negative results, especially in the setting of immunosuppression. Tuberculosis surveillance is therefore important during anti-TNF therapy because even after the initial screening (and latent tuberculosis treatment whenever appropriate), active disease can occur. If a patient develops tuberculosis, TNF antagonists should be discontinued and appropriate antituberculous therapy should be initiated ${ }^{[5]}$.

In conclusion, this clinical case highlights tuberculous tonsillitis as a consequence of anti-TNF therapy with adalimumab. It is important to stress this atypical presentation of tuberculosis in anti-TNF treated patients: the symptoms may mimic a conventional throat infection or even malignancy. Careful evaluation at the initiation of the treatment, as well as long-term surveillance of the patients receiving such drugs remains necessary.

\section{REFERENCES}

1. Armuzzi A, Lionetti P, Blandizzi C, Caporali R, Chimenti S, Cimino L, et al. Anti-TNF agents as therapeutic choice in immune-mediated inflammatory diseases: focus on adalimumab. Int J Immunopathol Pharmacol 2014;27:11-32.

2. Rifkin LM, Birnbaum AD, Goldstein DA. TNF inhibition for ophthalmic indications: current status and outlook. BioDrugs 2013;27:347-357.

3. Nacci F, Matucci-Cerinic M. Tuberculosis and other infections in the anti-tumour necrosis factor-alpha (anti-TNF- $\alpha$ ) era. Best Pract Res Clin Rheumatol 2011;25:375-388.

4. Sutbeyaz Y, Ucuncu H, Murat Karasen R, Gundogdu C. The association of secondary tonsillar and laryngeal tuberculosis: a case report and literature review. Auris Nasus Larynx 2000;27:371-374.

5. Carmona L, Gómez-Reino JJ, Rodríguez-Valverde V, Montero D, Pascual-Gómez E, Mola EM, et al. Effectiveness of recommendations to prevent reactivation of latent tuberculosis infection in patients treated with tumor necrosis factor antagonists. Arthritis Rheum 2005;52:1766-1772. 